\title{
A Special Case of Central Configuration for Planar Concave 5-body Problem with Newtonian Potentials
}

Yangshanshan LIU*

Department of Mathematics, Sichuan University, Chengdu 610064, P.R. China

DOI: $10.36347 /$ sipms.2020.v07i04.001

| Received: 19.04.2020 | Accepted: 27.04.2020 | Published: 30.04.2020

*Corresponding author: Yangshanshan LIU

Abstract

Original Research Article

In this paper we discuss a kind of symmetric configuration for planar concave 5-body problem with four of the five bodies on the vertex of an isosceles trapezoid and the other on the axis of symmetry inside the isosceles trapezoid. Then we give a special case to prove that there exist a set of positive masses to make this configuration a central configuration when the inner body not coincident with the center of mass.

Keywords: Central configurations, n-body problems, celestial mechanics.

Copyright @ 2020: This is an open-access article distributed under the terms of the Creative Commons Attribution license which permits unrestricted use, distribution, and reproduction in any medium for non-commercial use (NonCommercial, or CC-BY-NC) provided the original author and source are credited.

\section{INTRODUCTION}

From 1687 when Isaac Newton first proposed the 3-body problem in his great work Philosophice Naturalis Principia Mathematica [1], lots of excellent mathematicians in the history have been attracted to this direction. After the groundbreaking work of Henri Poincaré in the end of the 19th century and the beginning of the 20th century, the study of n-body problem has entered a new era, and many important results have been achieved though it's far from being done. Central configuration is of great significance in the study of this problem, and one of its core problem in this direction is that given any set of positive masses whether or not the classes of central configuration is finite. Naturally we can consider the inverse problem: given a configuration, under what conditions is it possible to choose positive masses that make it a central configuration. More details about N-body problem can be got in $[2,3,4,5,6,7,8,9,10,11,12,13]$.

For N-body problems, According to the Newton's law of motion and the law of gravitation, the i-th particle under the attaction of the other $\mathrm{N}-1$ particles satisfies the 2 rd differential equation.

$$
m_{k} \ddot{q}_{k}(t)=F_{k}=\sum_{j \neq k, j=1}^{N} \frac{G m_{j} m_{k}\left(q_{j}-q_{k}\right)}{\left|q_{j}-q_{k}\right|^{3}}=\frac{\partial U}{\partial q_{k}}, k=1, \ldots, N
$$

in which $\mathrm{G}$ is the gravitation constant, and

$$
U=\sum_{1 \leq k<j \leq N} \frac{G m_{j} m_{k}}{\left|q_{j}-q_{k}\right|}
$$

is the Newtonian potential.

The configuration set of this system collected by $\mathrm{N}$ particles is $\square^{d N}=\left\{q=\left(q_{1}, q_{2}, \ldots, q_{N}\right) \mid q_{k} \in \square^{d}, k=1,2, \ldots, N\right\}$ , while the collision set is $\Delta=\left\{q \in \square^{d N} \mid q_{j}=q_{k}, j \neq k\right\}$. Then we call the set $\square^{d N} \backslash \Delta$ a configuration space of the system. To simplify calculation we can choose appropriate dimension to make $G=1$.

Definition 1.1. $[14,15,16]$ If there exists $\lambda \in \square^{+}$to make the configuration $q \in \square^{d N} \backslash \Delta$ satifying

$$
-\lambda m_{k}\left(q_{k}-q_{c}\right)=\frac{\partial U(q)}{\partial q_{k}},
$$


or

$$
-\lambda m_{k}\left(q_{k}-q_{c}\right)=\sum_{j \neq k, j=1}^{N} \frac{G m_{j} m_{k}\left(q_{j}-q_{k}\right)}{\left|q_{j}-q_{k}\right|^{\beta}},
$$

then we call $q$ a central configuration.

The constant $\lambda$ is uniquely determined by $\lambda=\frac{U(q)}{I(q)}$. The center of mass is, $q_{c}=\frac{\sum_{k=1}^{N} m_{k} q_{k}}{\sum_{k=1}^{N} m_{k}}$, and the moment of inertia is $I(q)=\sum_{k=1}^{N} m_{k}\left|q_{k}-q_{c}\right|^{2}$

Specially, if we put the center of mass $q_{c}$ at the origin, the corresponding configuration space is $X \backslash \Delta$, where $X=\left\{q=\left(q_{1}, q_{2}, \ldots, q_{N}\right) \in \square^{d N} \mid q_{c}=0\right\}$.

In this paper, we study a special case of planar symmetric concave central configuration for 5-body problem with four of the five bodies on the vertex of an isosceles trapezoid and the other on the axis of symmetry inside the isosceles trapezoid.

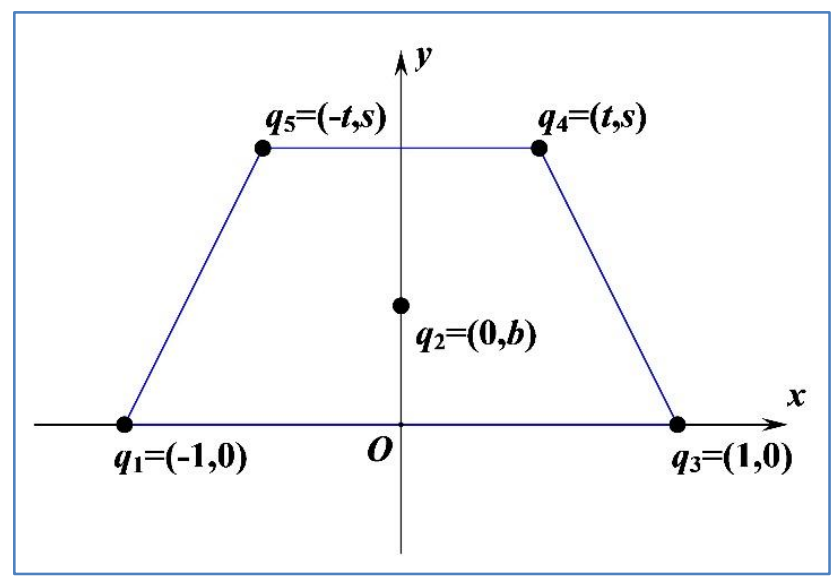

Fig-1: Isosceles trapezoid configuration

Now we establish the cartesian coordinate as shown in Figure 1, and the position of the five particles are $q_{1}=(-1,0), q_{2}=(0, b), q_{3}=(1,0), q_{4}=(t, s), q_{5}=(-t, s)$ Respectively as while as the center of mass $q_{c}=\left(c_{x}, c_{y}\right)$. By Definition 1.1, we write down the equations below.

$$
\left\{\begin{array}{l}
-\lambda\left(q_{1}-q_{c}\right)=m_{2} \frac{q_{2}-q_{1}}{\left|q_{2}-q_{1}\right|^{3}}+m_{3} \frac{q_{3}-q_{1}}{\left|q_{3}-q_{1}\right|^{3}}+m_{4} \frac{q_{4}-q_{1}}{\left|q_{4}-q_{1}\right|^{3}}+m_{5} \frac{q_{5}-q_{1}}{\left|q_{5}-q_{1}\right|^{3}} \\
-\lambda\left(q_{2}-q_{c}\right)=m_{1} \frac{q_{1}-q_{2}}{\left|q_{1}-q_{2}\right|^{3}}+m_{3} \frac{q_{3}-q_{2}}{\left|q_{3}-q_{2}\right|^{3}}+m_{4} \frac{q_{4}-q_{2}}{\left|q_{4}-q_{2}\right|^{3}}+m_{5} \frac{q_{5}-q_{2}}{\left|q_{5}-q_{2}\right|^{3}} \\
-\lambda\left(q_{3}-q_{c}\right)=m_{1} \frac{q_{1}-q_{3}}{\left|q_{1}-q_{3}\right|^{3}}+m_{2} \frac{q_{2}-q_{3}}{\left|q_{2}-q_{3}\right|^{3}}+m_{4} \frac{q_{4}-q_{3}}{\left|q_{4}-q_{3}\right|^{3}}+m_{5} \frac{q_{5}-q_{3}}{\left|q_{5}-q_{3}\right|^{3}} \\
-\lambda\left(q_{4}-q_{c}\right)=m_{1} \frac{q_{1}-q_{4}}{\left|q_{1}-q_{4}\right|^{3}}+m_{2} \frac{q_{2}-q_{4}}{\left|q_{2}-q_{4}\right|^{3}}+m_{3} \frac{q_{3}-q_{4}}{\left|q_{3}-q_{4}\right|^{3}}+m_{5} \frac{q_{5}-q_{4}}{\left|q_{5}-q_{4}\right|^{3}} \\
-\lambda\left(q_{5}-q_{c}\right)=m_{1} \frac{q_{1}-q_{5}}{\left|q_{1}-q_{5}\right|^{3}}+m_{2} \frac{q_{2}-q_{5}}{\left|q_{2}-q_{5}\right|^{3}}+m_{3} \frac{q_{3}-q_{5}}{\left|q_{3}-q_{5}\right|^{3}}+m_{4} \frac{q_{4}-q_{5}}{\left|q_{4}-q_{5}\right|^{3}}
\end{array}\right.
$$

Let $r_{i j}=\left|q_{i}-q_{j}\right|, i \neq j$, and with the symmetry of isosceles trapezoid we have $r_{12}=r_{23}, r_{24}=r_{25}, r_{14}=r_{35}, r_{15}=r_{34}$.

Simplify the equations above we can get 


$$
\left\{\begin{array}{l}
\lambda\left(c_{x}+1, c_{y}\right)=m_{2} \frac{(1, b)}{r_{12}^{3}}+m_{3} \frac{(2,0)}{r_{13}^{3}}+m_{4} \frac{(t+1, s)}{r_{14}^{3}}+m_{5} \frac{(1-t, s)}{r_{15}^{3}} \\
\lambda\left(c_{x}, c_{y}-b\right)=m_{1} \frac{(-1,-b)}{r_{12}^{3}}+m_{3} \frac{(1,-b)}{r_{12}^{3}}+m_{4} \frac{(t, s-b)}{r_{24}^{3}}+m_{5} \frac{(-t, s-b)}{r_{24}^{3}} \\
\lambda\left(c_{x}-1, c_{y}\right)=m_{1} \frac{(-2,0)}{r_{13}^{3}}+m_{2} \frac{(-1, b)}{r_{12}^{3}}+m_{4} \frac{(t-1, s)}{r_{15}^{3}}+m_{5} \frac{(-t-1, s)}{r_{14}^{3}} \\
\lambda\left(c_{x}-t, c_{y}-s\right)=m_{1} \frac{(-t-1,-s)}{r_{14}^{3}}+m_{2} \frac{(-t, b-s)}{r_{24}^{3}}+m_{3} \frac{(1-t,-s)}{r_{15}^{3}}+m_{5} \frac{(-2 t, 0)}{r_{45}^{3}} \\
\lambda\left(c_{x}+t, c_{y}-s\right)=m_{1} \frac{(t-1,-s)}{r_{15}^{3}}+m_{2} \frac{(t, b-s)}{r_{24}^{3}}+m_{3} \frac{(t+1,-s)}{r_{14}^{3}}+m_{4} \frac{(2 t, 0)}{r_{45}^{3}}
\end{array}\right.
$$

Divide (0.6) equation reference goes here into two parts we get

$$
\left\{\begin{array}{l}
\lambda\left(c_{x}+1\right)=m_{2} \frac{1}{r_{12}^{3}}+m_{3} \frac{2}{r_{13}^{3}}+m_{4} \frac{t+1}{r_{14}^{3}}+m_{5} \frac{-t+1}{r_{15}^{3}} \\
\lambda c_{x}=m_{1} \frac{-1}{r_{12}^{3}}+m_{3} \frac{1}{r_{12}^{3}}+m_{4} \frac{t}{r_{24}^{3}}+m_{5} \frac{-t}{r_{24}^{3}} \\
\lambda\left(c_{x}-1\right)=m_{1} \frac{-2}{r_{13}^{3}}+m_{2} \frac{-1}{r_{12}^{3}}+m_{4} \frac{t-1}{r_{15}^{3}}+m_{5} \frac{-t-1}{r_{14}^{3}} \\
\lambda\left(c_{x}-t\right)=m_{1} \frac{-t-1}{r_{14}^{3}}+m_{2} \frac{-t}{r_{24}^{3}}+m_{3} \frac{-t+1}{r_{15}^{3}}+m_{5} \frac{-2 t}{r_{45}^{3}} \\
\lambda\left(c_{x}+t\right)=m_{1} \frac{t-1}{r_{15}^{3}}+m_{2} \frac{t}{r_{24}^{3}}+m_{3} \frac{t+1}{r_{14}^{3}}+m_{4} \frac{2 t}{r_{45}^{3}} \\
\lambda\left(c_{y}-b\right)=m_{2} \frac{b}{r_{12}^{3}}+m_{4} \frac{s}{r_{14}^{3}}+m_{5} \frac{s}{r_{15}^{3}}+m_{3} \frac{-b}{r_{12}^{3}}+m_{4} \frac{s-b}{r_{24}^{3}}+m_{5} \frac{s-b}{r_{24}^{3}} \\
\lambda c_{y}=m_{2} \frac{b}{r_{12}^{3}}+m_{4} \frac{s}{r_{15}^{3}}+m_{5} \frac{s}{r_{14}^{3}} \\
\lambda\left(c_{y}-s\right)=m_{1} \frac{-s}{r_{14}^{3}}+m_{2} \frac{b-s}{r_{24}^{3}}+m_{3} \frac{-s}{r_{15}^{3}} \\
\lambda\left(c_{y}-s\right)=m_{1} \frac{-s}{r_{15}^{3}}+m_{2} \frac{b-s}{r_{24}^{3}}+m_{3} \frac{-s}{r_{14}^{3}}
\end{array}\right.
$$

In (0.8), subtracting the first formula from the third formula and the fourth from the fifth we have $0=\left(m_{4}-m_{5}\right)\left(\frac{s}{r_{14}^{3}}-\frac{s}{r_{15}^{3}}\right)$ and $0=\left(m_{1}-m_{3}\right)\left(\frac{s}{r_{15}^{3}}-\frac{s}{r_{14}^{3}}\right)$.

For $r_{14}=\sqrt{(t+1)^{2}+s^{2}} \neq \sqrt{(t-1)^{2}+s^{2}}=r_{15}, 0<t \leq 1$, we have $m_{1}=m_{3}, m_{4}=m_{5}$.

Substituting (0.9) into the second formula in (0.7) we have

$$
c_{x}=0
$$

Then from (0.9) and (0.10) we can simplify (0.7) and (0.8) as below 


$$
\left\{\begin{array}{l}
\lambda=m_{1} \frac{2}{r_{13}^{3}}+m_{2} \frac{1}{r_{12}^{3}}+m_{4}\left(\frac{t+1}{r_{14}^{3}}+\frac{-t+1}{r_{15}^{3}}\right) \\
\lambda t=m_{1}\left(\frac{t-1}{r_{15}^{3}}+\frac{t+1}{r_{14}^{3}}\right)+m_{2} \frac{t}{r_{24}^{3}}+m_{4} \frac{2 t}{r_{45}^{3}} \\
\lambda c_{y}=m_{2} \frac{b}{r_{12}^{3}}+m_{4}\left(\frac{s}{r_{14}^{3}}+\frac{s}{r_{15}^{3}}\right) \\
\lambda\left(c_{y}-b\right)=m_{1} \frac{-2 b}{r_{12}^{3}}+m_{4} \frac{2(s-b)}{r_{24}^{3}} \\
\lambda\left(c_{y}-s\right)=m_{1}\left(\frac{-s}{r_{14}^{3}}+\frac{-s}{r_{15}^{3}}\right)+m_{2} \frac{b-s}{r_{24}^{3}}
\end{array}\right.
$$

\section{RESULTS AND DISCUSSION}

Theorem 2.1. If $q_{2}$ is not coincident with the center of mass $q_{c}$, i.e. $q_{2} \neq q_{c}$, and if $t=0.5$, then there exists regions $G$ and $K$ (Figure $\underline{1}$ ) in the $b-s$ plane to make the configuration a central configuration.

Firstly by carefully calculating and simplifying we can get the fomulas below from $(0.11)$

$$
\begin{gathered}
m_{1}=\lambda \cdot(s-b) \cdot \frac{\left(\frac{1}{r_{12}^{3}}-\frac{1}{r_{24}^{3}}\right)\left(\frac{2(s-b)}{r_{24}^{3}}+\frac{b(t+1)-s}{r_{14}^{3}}+\frac{b(-t+1)-s}{r_{15}^{3}}\right)}{\left(\frac{-2(s-b)}{r_{24}^{3}}-\frac{2 b}{r_{12}^{3}}+\frac{s}{r_{14}^{3}}+\frac{s}{r_{15}^{3}}\right)\left(\frac{2(s-b)}{r_{13}^{3} r_{24}^{3}}+\frac{b(t+1)-s}{r_{12}^{3} r_{14}^{3}}+\frac{b(-t+1)-s}{r_{12}^{3} r_{15}^{3}}\right)}, \\
m_{2}=\lambda \cdot \frac{Q}{\left(\frac{2(s-b)}{r_{13}^{3} r_{24}^{3}}+\frac{b(t+1)-s}{r_{12}^{3} r_{14}^{3}}+\frac{b(-t+1)-s}{r_{12}^{3} r_{15}^{3}}\right)\left(\frac{-2(s-b)}{r_{24}^{3}}-\frac{2 b}{r_{12}^{3}}+\frac{s}{r_{14}^{3}}+\frac{s}{r_{15}^{3}}\right)}, \\
m_{4}=\lambda \cdot \frac{2 b(s-b)\left(\frac{1}{r_{24}^{3}}-\frac{1}{r_{12}^{3}}\right)\left(\frac{1}{r_{13}^{3}}-\frac{1}{r_{12}^{3}}\right)}{\left(\frac{-2(s-b)}{r_{24}^{3}}-\frac{2 b}{r_{12}^{3}}+\frac{s}{r_{14}^{3}}+\frac{s}{r_{15}^{3}}\right)\left(\frac{2(s-b)}{r_{13}^{3} r_{24}^{3}}+\frac{b(t+1)-s}{r_{12}^{3} r_{14}^{3}}+\frac{b(-t+1)-s}{r_{12}^{3} r_{15}^{3}}\right)},
\end{gathered}
$$

where

$$
\begin{aligned}
Q= & \frac{2 s(s-b)}{r_{14}^{3} r_{24}^{3}}+\frac{2 s(s-b)}{r_{15}^{3} r_{24}^{3}}+\frac{-4 s(s-b)}{r_{13}^{3} r_{24}^{3}}+\frac{-2 b s t}{r_{12}^{3} r_{14}^{3}}+\frac{2 b s t}{r_{12}^{3} r_{15}^{3}} \\
& +\frac{2 s(s-b)}{r_{13}^{3} r_{14}^{3}}+\frac{2 s(s-b)}{r_{13}^{3} r_{15}^{3}}+\frac{s(b(t+1)-s)}{r_{14}^{6}}+\frac{-2 s(s-b)}{r_{14}^{3} r_{15}^{3}}+\frac{s(b(-t+1)-s)}{r_{15}^{6}},
\end{aligned}
$$

and the equation of $t, b, s$

$$
\begin{aligned}
& \frac{2 b t(b(t+1)-s)}{r_{12}^{3} r_{14}^{3}}+\frac{2 b t(b(-t+1)-s)}{r_{12}^{3} r_{15}^{3}}+\frac{4 b t(s-b)}{r_{12}^{3} r_{45}^{3}}+\frac{4 b t(s-b)}{r_{13}^{3} r_{24}^{3}}+\frac{(b(t-1)+s)^{2}}{r_{15}^{6}} \\
& =\frac{4 b t(s-b)}{r_{13}^{3} r_{45}^{3}}+\frac{(b(t+1)-s)^{2}}{r_{14}^{6}}+\frac{2(s-b)(b(t+1)-s)}{r_{14}^{3} r_{24}^{3}}+\frac{2(s-b)(b(t-1)+s)}{r_{15}^{3} r_{24}^{3}}
\end{aligned}
$$

With (0.16) we can get some new notifications as below

As while as

$$
\begin{aligned}
& a_{1}=\left(\frac{-2(s-b)}{r_{24}^{3}}-\frac{2 b}{r_{12}^{3}}+\frac{s}{r_{14}^{3}}+\frac{s}{r_{15}^{3}}\right) \\
& a_{2}=\left(\frac{2(s-b)}{r_{13}^{3} r_{24}^{3}}+\frac{b(t+1)-s}{r_{12}^{3} r_{14}^{3}}-\frac{b(t-1)+s}{r_{12}^{3} r_{15}^{3}}\right), \\
& a_{3}=\left(\frac{2(s-b)}{r_{24}^{3}}+\frac{b(t+1)-s}{r_{14}^{3}}-\frac{b(t-1)+s}{r_{15}^{3}}\right)
\end{aligned}
$$


Where

$$
\begin{aligned}
Q & =-2 s a_{2}+s a_{3}\left(\frac{1}{r_{14}^{3}}+\frac{1}{r_{15}^{3}}\right)+2 s(s-b)\left(\frac{1}{r_{12}^{3}}-\frac{1}{r_{13}^{3}}\right)\left(\frac{1}{r_{14}^{3}}+\frac{1}{r_{15}^{3}}\right) \\
& =\frac{s(s-b)}{b t}\left[\left(\frac{1}{r_{14}^{3}}-\frac{1}{r_{15}^{3}}\right) a_{3}+2 b t\left(\frac{1}{r_{12}^{3}}-\frac{1}{r_{13}^{3}}\right)\left(\frac{1}{r_{14}^{3}}+\frac{1}{r_{15}^{3}}+\frac{2}{r_{45}^{3}}\right)\right] \\
& =\frac{s(s-b)}{b t} Q_{1}
\end{aligned}
$$

$$
Q_{1}=\left(\frac{1}{r_{14}^{3}}-\frac{1}{r_{15}^{3}}\right) a_{3}+2 b t\left(\frac{1}{r_{12}^{3}}-\frac{1}{r_{13}^{3}}\right)\left(\frac{1}{r_{14}^{3}}+\frac{1}{r_{15}^{3}}+\frac{2}{r_{45}^{3}}\right)
$$

Then we define

$$
\begin{aligned}
& c_{1}=\sqrt{3}-b \\
& c_{2}=t^{2}+(s-b)^{2}-b^{2}-1 \\
& c_{3}=s-b
\end{aligned}
$$

And from simple factorization we easily know that $r_{13}^{2}-r_{12}^{2}=3-b^{2}, r_{24}^{2}-r_{12}^{2}=t^{2}+(s-b)^{2}-b^{2}-1$, so $c_{1}$ and $\sqrt{3}-b, c_{2}$ and $t^{2}+(s-b)^{2}-b^{2}-1$ share the same sign respectively.

Then we get seven new functions $a_{1}, a_{2}, a_{3}, Q_{1}, c_{1}, c_{2}, c_{3}$ with variables $b$ and $s$.

Secondly we analysis the necessary and sufficient condition which makes $m_{1}, m_{2}, m_{4}$ all positive

1) $a_{1} a_{2}>0$ :

$$
(1-1)\left\{\begin{array} { l } 
{ Q _ { 1 } > 0 } \\
{ a _ { 3 } > 0 } \\
{ c _ { 1 } > 0 } \\
{ c _ { 2 } > 0 }
\end{array} , \quad \text { or } \quad ( 1 - 2 ) \left\{\begin{array}{l}
Q_{1}>0 \\
a_{3}<0 \\
c_{1}<0 \\
c_{2}<0
\end{array}\right.\right.
$$

2) $\quad a_{1} a_{2}<0$ :

$$
(2-1)\left\{\begin{array} { l } 
{ Q _ { 1 } < 0 } \\
{ a _ { 3 } < 0 } \\
{ c _ { 1 } < 0 } \\
{ c _ { 2 } > 0 }
\end{array} , \quad \text { or } \quad ( 2 - 2 ) \left\{\begin{array}{l}
Q_{1}<0 \\
a_{3}>0 \\
c_{1}>0 \\
c_{2}<0
\end{array}\right.\right.
$$

Thirdly, let $t=0.5, b \in(0,2.5), s \in(0,4)$, and by using computer we can get the regions that make all $m_{i}>0(i=1,2,4)$ positive (see Figure 2).

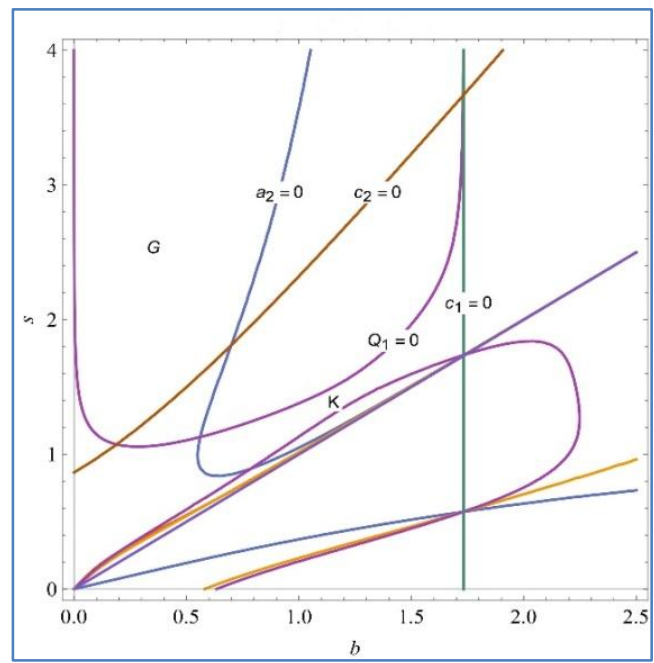

Fig-2: The final regions in plane $b-s$

Finally, we list a table to illustrate the shape of central configuration in region $G$ and region $K$. 
Table-1: Cases of Central configruations in $G, K$

\begin{tabular}{|c|c|c|c|c|c|}
\hline$t=0.5$ & $m_{1}=m_{3}$ & $m_{2}$ & $m_{4}=m_{5}$ & $c_{y}$ & Figure \\
\hline$(0.3,1.5) \in G$ & $1.1559 \lambda$ & $18.6558 \lambda$ & $0.8156 \lambda$ & 0.5816 & Fig 3 \\
\hline$(1.6,2) \in K$ & $3.7249 \lambda$ & $0.0554 \lambda$ & $0.0969 \lambda$ & 0.1208 & Fig 4 \\
\hline
\end{tabular}

Keep the four decimal places.

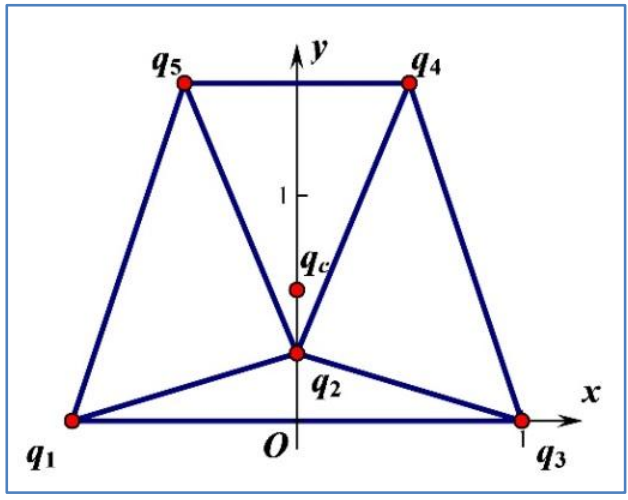

Fig-3: Central configruations in $G$

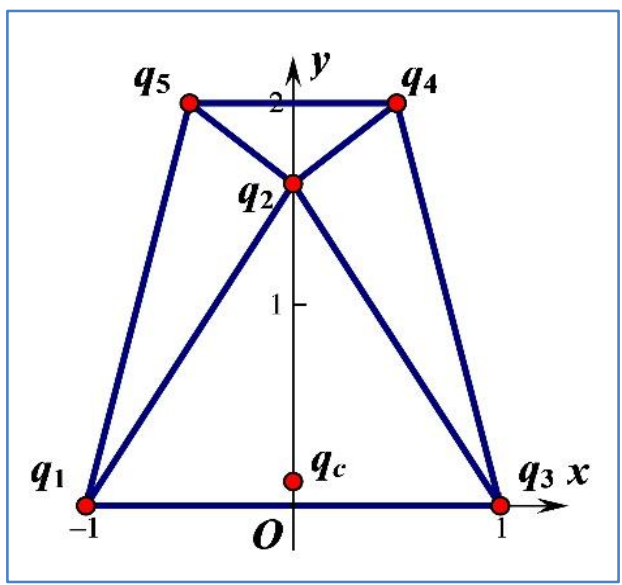

Fig-4: Central configruations in $K$

\section{ACKNOWLEDGMENT}

The author would like to thank Prof. Zhang Shiqing for his instructive and suggestions.

\section{REFERENCES}

1. Isaac Newton. Philosophiae naturalis principia mathematica. Philosophiae naturalis principia mathematica, 2016.

2. Chunhua Deng and Shiqing Zhang. Planar symmetric concave central configurations in newtonian four-body problems. Journal of Geometry and Physics. 83:43-52, 2014.

3. Yiming Long. Admissible shapes of 4-body noncollinear relative equilibria. Advanced Nonlinear Studies. 3(4):495-509, 2003.
4. Zhifu Xie. Inverse problem of central configurations and singular curve in the collinear 4body problem. Celestial Mechanics and Dynamical Astronomy. 107(3):353-376, 2010.

5. Yiming Long and Shanzhong Sun. Four-body central configurations with some equal masses. Archive for rational mechanics and analysis. 162(1):25-44, 2002.

6. Jaume Llibre and Claudia Valls. The co-circular central configurations of the 5-body problem. Journal of Dynamics and Differential Equations. 27(1):55-67, 2015.

7. Marshall Hampton and Anders Jensen. Finiteness of spatial central configurations in the five-body problem. Celestial Mechanics and Dynamical Astronomy. 109(4):321-332, 2011.

8. Martha Alvarez-Ramrez, Montserrat Corbera, and Jaume Llibre. Ón the central configurations in the spatial 5-body problem with four equal masses. Celestial Mechanics and Dynamical Astronomy. 124(4):433-456, 2016.

9. Muhammad Shoaib, Abdul Rehman Kashif, and Iharka Szücs-Csillik. On the planar central configurations of rhomboidal and triangular fourand five-body problems. Astrophysics and Space Science. 362(10):182, 2017.

10. J Lino Cornelio, Martha Álvarez-Ramrez, and Josep M Cors. Á family of stacked central configurations in the planar five-body problem. Celestial Mechanics and Dynamical Astronomy. 129(3):321-328, 2017.

11. Alain Albouy and Richard Moeckel. The inverse problem for collinear central configurations. Celestial Mechanics and Dynamical Astronomy. 77(2):77-91, 2000.

12. Forest Ray Moulton. The straight line solutions of the problem of $\mathrm{n}$ bodies. The Annals of Mathematics. 12(1):1-17, 1910.

13. Aurel Wintner. The Analytical Foundations of Celestial Mechanics; 2014.

14. Richard Moeckel. On central configurations. Mathematische Zeitschrift. 205(1):499-517, 1990.

15. Richard Montgomery. The three-body problem and the shape sphere. The American Mathematical Monthly, 122(4):299-321, 2015.

16. Vladimir I Arnold, VV Kozlov, and AI Neishtadt. Dynamical systems III. Springer; 1988. 